CARPATHIAN J. MATH.

Volume 37 (2021), No. 3,

Pages 497 - 512
Online version at https : //www . carpathian. cunbm . utcluj. ro/

Print Edition: ISSN 1584 - 2851; Online Edition: ISSN 1843 - 4401

DOI: https://doi.org/10.37193/CJM.2021.03.12

\title{
Iterative methods for optimization problems and image restoration
}

\author{
AnANTACHAi Padcharoen and DuAngKamon KitKuAN
}

ABSTRACT. In this paper, we introduce a new accelerated iterative method for finding a common fixed point of a countable family of nonexpansive mappings in the Hilbert spaces framework. Using our main result, we obtain a new accelerated image restoration iterative method for solving a minimization problem in the form of the sum of two proper lower semi-continuous and convex functions. As applications, we apply our algorithm to solving image restoration problems.

\section{INTRODUCTION}

Optimization theory is widely used as it can be used to solve many practical problems such as engineering, economics, computer science, and applied science. The study of optimization theory is based on functional analysis, nonlinear analysis, and convex analysis. From an operational perspective, numerical analysis was considered to solve the optimization problem and to show the convergence of the sequence. It is studied or solved using numerical iterative methods, most of which are used in everyday life.

At present, there is various photography. Be it from a camera or a scan, perhaps a blurry image, such as a blurry, blurred, or dark spot. That makes the picture distort the truth as in the Figure 1.

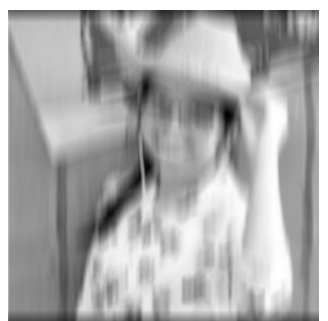

(A) Kitkuan

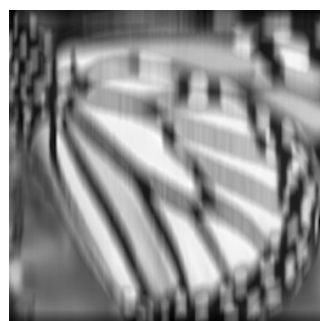

(B) Butterfly

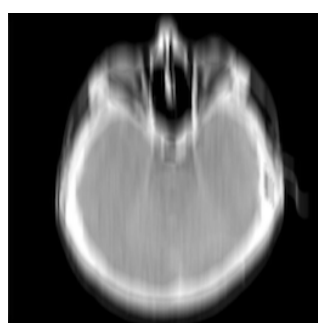

(C) HeadCT

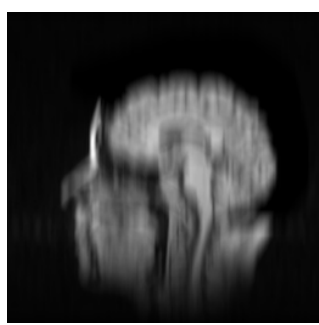

(D) Brain

FIGURE 1. Test images 2.

From the above problem can be written in mathematical models as shown in the Figure

Received: 05.10.2020. In revised form: 16.04.2021. Accepted: 23.04.2021

2010 Mathematics Subject Classification. 47H09, 47H10, 54H25.

Key words and phrases. Convex minimization, proximal mapping technique, Hilbert space, approximation method, optimization problems, image restoration.

Corresponding author: Duangkamon Kitkuan; or_duangkamon@hotmail.com 


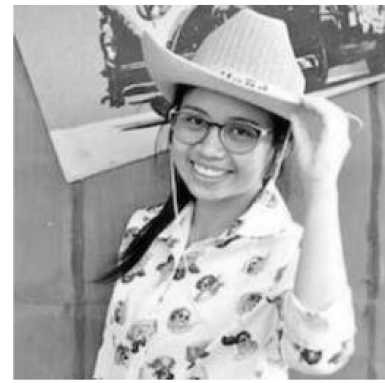

$\mathbf{x}(\mathbf{i}, \mathbf{j})$

original image

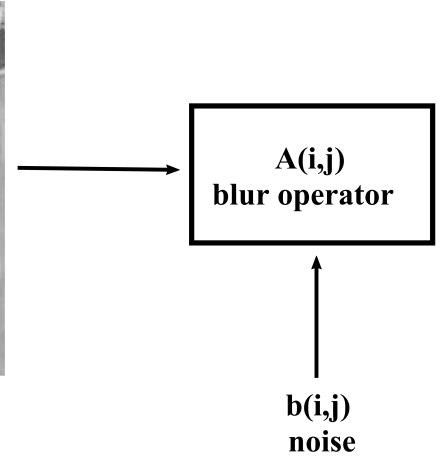

noise

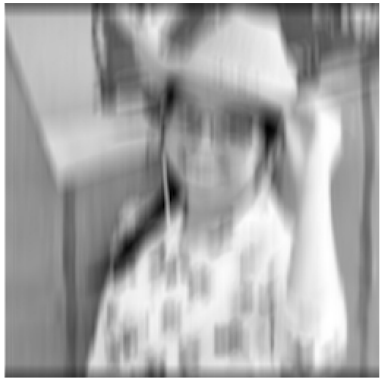

$\mathbf{h}(\mathbf{i}, \mathbf{j})$

degraded image

FIGURE 2. Degradation model

We can be written as an equation as follows:

$$
h=A x+b,
$$

where $x \in \mathbb{R}^{n}$ is original image, $A$ is blur operator, $b$ is noise, and $h$ is degraded image. In order to solve the problem (1.1), Tibshirani in [19], introduced the least absolute shrinkage and selection operator (LASSO) for solving the following minimization problem:

$$
\min _{x} \frac{1}{2}\|A x-h\|_{2}^{2}+\mu\|x\|_{1}
$$

where $\mu>0$ is a regularization parameter, $\|x\|_{1}=\sum_{i=1}^{n}\left|x_{i}\right|$ and $\|x\|_{2}=\sqrt{\sum_{i=1}^{n}\left|x_{i}\right|^{2}}$. In the theory of optimal optimization, concrete problems can be solved in many ways:

$$
\begin{aligned}
& \operatorname{minimize} f(x)+g(x) \\
& \text { subject to } x \in \mathbb{R}^{n},
\end{aligned}
$$

where $f: \mathbb{R}^{n} \rightarrow \mathbb{R}$ is a convex smooth function and $g: \mathbb{R}^{n} \rightarrow \mathbb{R} \cup\{\infty\}$ is a proper convex lower semi-continuous and nonsmooth function. The solution of (1.3) can be characterized by Theorem 16.3 of Bauschke and Combettes [1] as follows:

$$
w \text { is a minimizer of }(f+g) \text { if and only if } 0 \in \partial g(w)+\nabla f(w),
$$

where $\partial g$ is subdifferential of $g$ and $\nabla f$ is the gradient of $f$. The subdifferential of $g$ at $w$, denoted by $\partial g(w)$, is defined by

$$
\partial g(w):=\{z: g(x) \geq\langle z, x-w\rangle+g(w) \text { for all } x\} .
$$

It is well-known that the subdifferential operator $\partial g$ is maximal monotone, see [3] for more details. For solving (1.3) is characterized by the following fixed point problem:

$$
w \text { is a minimizer of }(f+g) \text { if and only if } w=\underset{r g}{\operatorname{prox}}(w-r \nabla f(w)),
$$

for any $r>0$ and prox $_{r g}$ is the proximity operator of $g$ defined by

$$
\operatorname{prox}_{g}(x)=\underset{z}{\arg \min }\left\{g(z)+\frac{\|x-z\|^{2}}{2}\right\} .
$$

Moudafi and Oliny [14] introduced a method called Inertial method, which is another name for the heavy ball method, as follows:

$$
\left\{\begin{array}{l}
z_{n}=x_{n}+\theta_{n}\left(x_{n}-x_{n-1}\right), \\
x_{n+1}=\operatorname{prox}_{r g}\left(z_{n}-r_{n} \nabla f\left(x_{n}\right)\right), \quad \forall n \geq 1
\end{array}\right.
$$


and proved that this algorithm converts to the minimum of $f+g$ which $r_{n}<\frac{1}{L}$, where $L$ is the lipchitz constant of $\nabla f$.

Beck and Teboulle [2] introduced a so-called FISTA (fast iterative shrinkage-thresholding algorithm) as follows:

$$
\left\{\begin{array}{l}
x_{1}=z_{0}, t_{0}=1 \\
z_{n}=\operatorname{prox}_{r_{n} g}\left(x_{n}-r_{n} \nabla f\left(x_{n}\right)\right) \\
t_{n+1}=\frac{1+\sqrt{4 t_{n}^{2}+1}}{2} \\
\theta_{n}=\frac{t_{n}-1}{t_{n+1}}, \\
x_{n+1}=z_{n}+\theta_{n}\left(z_{n}-z_{n-1}\right), \quad \forall n \geq 1 .
\end{array}\right.
$$

Recently, Verma and Shukla [22] introduced a new accelerated proximal gradient algorithm (NAGA) as follow:

$$
\left\{\begin{array}{l}
z_{n}=x_{n}+\theta_{n}\left(x_{n}-x_{n-1}\right), \\
x_{n+1}=T_{n}\left(\left(1-\delta_{n}\right) z_{n}+\delta_{n} T_{n} z_{n}\right), \quad \forall n \geq 1,
\end{array}\right.
$$

where $x_{0}, x_{1} \in \mathbb{R}^{n}, T_{n}$ is the forward-backward operator of $f$ and $g$ with respect to $r_{n} \in(0,2 / L)$. They proved the convergence of the NAGA and applied to solving the convex minimization problem with sparsity-inducing regularizes for multitask learning framework.

Motivated by those works mentioned above, in this paper, a new accelerated fixed point algorithms for solving (1.3) by Ishikawa type with the inertial step for a countable family of nonexpansive mappings.

\section{PRELIMINARIES}

The fundamentals of the Hilbert space are studied, as well as definitions and theorems. It is used to prove it as follows:

Definition 2.1. [19] The two real-value functions $\langle\cdot, \cdot\rangle: X \times X \rightarrow \mathbb{R}$ are called the inner product (inner product) on the $X$ vector space for members $x, y, z \in X$ and the constants $\alpha, \beta \in \mathbb{R}$ satisfy the following conditions

(i) $\langle x, x\rangle \geq 0$ for all $x \in X$;

(ii) $\langle x, x\rangle=0$ if and only if $x=0$;

(iii) $\langle x, y\rangle=\langle y, x\rangle$;

(iv) $\langle\alpha x+\beta y, z\rangle=\alpha\langle x, z\rangle+\beta\langle y, z\rangle$.

The vector space $X$ and the internal product $\langle\cdot, \cdot\rangle$ can be written together $(X,\langle\cdot, \cdot\rangle)$, which is called the inner product space.

In addition, various features of the convergence sequence in the Hilbert space $\mathcal{H}$ are investigated, beginning with the naming and proof of the following theorem.

Lemma 2.1. [19] Let $\mathcal{H}$ be a Hilbert space. The following statement holds in $\mathcal{H}$ :

$$
\|\alpha x+\rho z\|^{2}=\alpha(\alpha+\rho)\|x\|^{2}+\rho(\alpha+\rho)\|z\|^{2}-\rho \alpha\|x-z\|^{2}, \forall x, z \in \mathcal{H}, \forall \alpha, \rho \in \mathbb{R} .
$$

Let $\mathcal{H}$ be a real Hilbert space with norm $\|\cdot\|$ and inner product $\langle\cdot, \cdot\rangle$, and $C$ be a nonempty closed convex subset of $\mathcal{H}$.

A nonlinear operator $T: C \rightarrow C$ is called

(i) $L$-Lipschitz operator, if there exists $L>0$ such that

$$
\|T x-T z\| \leq L\|x-z\|, \quad \text { for all } x, z \in C ;
$$


(ii) nonexpansive, if

$$
\|T x-T z\| \leq\|x-z\|, \quad \text { for all } x, z \in C .
$$

Next, we denote by Fix $(T)$ the set of all fixed points of $T, F i x(T):=\{w \in C: T w=$ $w\}, \omega_{w}\left(x_{n}\right)$ denote the set of all weak-cluster points of a bounded sequence $\left\{x_{n}\right\}$ in $C,\left\{T_{n}\right\}$ and $\Psi$ be families of nonexpansive operators of $C$ into itself such that $\Omega:=$ $\bigcap_{k=1}^{\infty} \operatorname{Fix}\left(T_{n}\right) \supset \operatorname{Fix}(\Psi) \neq \emptyset$, where $\operatorname{Fix}(\Psi)$ is the set of all common fixed points of $\Psi$.

Nakajo et al. [15] introduced the NST-condition (I) with $\Psi$. A sequence $\left\{T_{n}\right\}$ is said to satisfy the NST if for every bounded sequence $\left\{x_{n}\right\}$ in $C$,

$$
\lim _{n \rightarrow \infty}\left\|x_{n}-T_{n} x_{n}\right\|=0 \quad \text { implies } \quad \lim _{n \rightarrow \infty}\left\|x_{n}-T x_{n}\right\|=0, \quad \forall T \in \Psi .
$$

Nakajo et al. [16] introduced the NST*-condition which is more general than that of NSTcondition. A sequence $\left\{T_{n}\right\}$ is said to satisfy the NST*-condition if for every bounded sequence $\left\{x_{n}\right\}$ in $C$,

$$
\lim _{n \rightarrow \infty}\left\|x_{n}-T_{n} x_{n}\right\|=\lim _{n \rightarrow \infty}\left\|x_{n}-x_{n+1}\right\|=0 \quad \text { implies } \quad \omega_{w}\left(x_{n}\right) \subset \Omega .
$$

Lemma 2.2. [14] Let $\mathcal{H}$ be a Hilbert space and $\left\{x_{n}\right\}$ be a sequence in $\mathcal{H}$ such that there exists a nonempty set $\Omega \subset \mathcal{H}$ satisfying

(i) for every $x \in \Omega, \lim _{n \rightarrow \infty}\left\|x_{n}-x\right\|$ exists;

(ii) each weak-cluster point of the sequence $\left\{x_{n}\right\}$ is in $\Omega$.

Then there exists $w \in \Omega$ such that $\left\{x_{n}\right\}$ weakly converges to $w$.

Lemma 2.3. [4] For a real Hilbert space $\mathcal{H}$, let $g: \mathcal{H} \rightarrow \mathbb{R} \cup\{\infty\}$ be a proper convex and lower semi-continuous function, and $f: \mathcal{H} \rightarrow \mathbb{R}$ be convex differentiable with gradient $\nabla f$ being $L$-Lipschitz constant for some $L>0$. If $\left\{T_{n}\right\}$ is the forward-backward operator of $f$ and $g$ with respect to $r_{n} \in(0,2 / L)$ such that $r_{n}$ converges to $r$, then $\left\{T_{n}\right\}$ satisfies NST-condition (I) with $T$, where $T$ is the forward-backward operator of $f$ and $g$ with respect to $r \in(0,2 / L)$.

Lemma 2.4. [11] Let $\left\{v_{n}\right\},\left\{\delta_{n}\right\}$ and $\left\{\theta_{n}\right\}$ are sequence in $[0, \infty)$ such that

$$
v_{n+1} \leq v_{n}+\theta_{n}\left(v_{n}-v_{n-1}\right)+\delta_{n}, \forall n \geq 1, \sum_{n=1}^{\infty} \delta_{n}<\infty
$$

and there exists $\theta$ which $0 \leq \theta_{n} \leq \theta<1$ for all $n \in \mathbb{N}$.

So the following statement hold:

(i) $\sum_{n=1}^{\infty}\left[v_{n}-v_{n-1}\right]_{+}<\infty$, where $[t]_{+}:=\max \{t, 0\}$;

(ii) there exists $v^{*} \in[0, \infty)$, where $\lim _{n \rightarrow \infty} v_{n}=v^{*}$.

\section{MAIN RESULTS}

Study of the convergence theory of repeat methodology. To the answer to the problem of optimal optimization in the Hilbert space $\mathcal{H}$ and from the theorem constructions and improvements. Obtain important new knowledge with key content as follows:

Theorem 3.1. Let $\left\{T_{n}: \mathcal{H} \rightarrow \mathcal{H}\right\}$ be a family of nonexpansive operators. Suppose $\left\{T_{n}\right\}$ satisfies $N S T^{*}$-condition and $\Omega:=\bigcap_{n=1}^{\infty} F i x\left(T_{n}\right) \neq \emptyset$. Let the sequence $\left\{x_{n}\right\}$ in $\mathcal{H}$ be generated by choosing $x_{0}, x_{1} \in \mathcal{H}$ and using the recursion

$$
\left\{\begin{array}{l}
w_{n}=x_{n}+\theta_{n}\left(x_{n}-x_{n-1}\right), \\
z_{n}=\left(1-\tau_{n}\right) x_{n}+\tau_{n}\left(\left(1-\gamma_{n}\right) w_{n}+\gamma_{n} T_{n} w_{n}\right), \\
x_{n+1}=\left(1-\delta_{n}\right) x_{n}+\delta_{n}\left(\left(1-\lambda_{n}\right) z_{n}+\lambda_{n} T_{n} z_{n}\right),
\end{array}\right.
$$

where $\left\{\tau_{n}\right\},\left\{\gamma_{n}\right\},\left\{\delta_{n}\right\},\left\{\lambda_{n}\right\}$ and $\left\{\theta_{n}\right\}$ are sequences such that 
(i) $0 \leq \theta_{n} \leq \theta_{n+1} \leq 1$;

(ii) $0<\tau \leq \tau_{n} \leq \tau_{n+1} \leq \frac{1}{2+\alpha}:=\epsilon, \alpha>0$;

(iii) $0<\gamma \leq \gamma_{n} \leq \rho<1$;

(iv) $0<\delta \leq \delta_{n} \leq \delta_{n+1} \leq \frac{1}{2+\beta}:=\varepsilon, \beta>0$;

(v) $0<\lambda \leq \lambda_{n} \leq \iota<1$.

Then the sequence $\left\{x_{n}\right\}$ generated by (3.7) converges weakly to a point $w \in \Omega$.

Proof. Let $w \in \Omega, \quad \zeta_{n}=\left(1-\gamma_{n}\right) w_{n}+\gamma_{n} T_{n} w_{n}$ and $\vartheta_{n}=\left(1-\lambda_{n}\right) z_{n}+\lambda_{n} T_{n} z_{n}$. Using Lemma 2.1, we obtain

$$
\begin{aligned}
\left\|\zeta_{n}-w\right\|^{2}= & \left\|\left(1-\gamma_{n}\right) w_{n}+\gamma_{n} T_{n} w_{n}-w\right\|^{2} \\
= & \left\|\left(1-\gamma_{n}\right)\left(w_{n}-w\right)+\gamma_{n}\left(T_{n} w_{n}-w\right)\right\|^{2} \\
= & \left(1-\gamma_{n}\right)\left\|w_{n}-w\right\|^{2}+\gamma_{n}\left\|T_{n} w_{n}-w\right\|^{2} \\
& -\gamma_{n}\left(1-\gamma_{n}\right)\left\|T_{n} w_{n}-w_{n}\right\|^{2} \\
\leq & \left\|w_{n}-w\right\|^{2}-\gamma_{n}\left(1-\gamma_{n}\right)\left\|T_{n} w_{n}-w_{n}\right\|^{2} .
\end{aligned}
$$

Hence,

$$
\left\|\zeta_{n}-w\right\| \leq\left\|w_{n}-w\right\|
$$

and

$$
\begin{aligned}
\left\|\vartheta_{n}-w\right\|^{2}= & \left\|\left(1-\lambda_{n}\right) z_{n}+\lambda_{n} T_{n} z_{n}-w\right\|^{2} \\
= & \left\|\left(1-\lambda_{n}\right)\left(z_{n}-w\right)+\lambda_{n}\left(T_{n} z_{n}-w\right)\right\|^{2} \\
= & \left(1-\lambda_{n}\right)\left\|z_{n}-w\right\|^{2}+\lambda_{n}\left\|T_{n} z_{n}-w\right\|^{2} \\
& -\lambda_{n}\left(1-\lambda_{n}\right)\left\|T_{n} z_{n}-z_{n}\right\|^{2} \\
\leq & \left\|z_{n}-w\right\|^{2}-\lambda_{n}\left(1-\lambda_{n}\right)\left\|T_{n} z_{n}-z_{n}\right\|^{2} .
\end{aligned}
$$

So,

$$
\left\|\vartheta_{n}-w\right\| \leq\left\|z_{n}-w\right\| .
$$

From (3.9), we get

$$
\begin{aligned}
\left\|x_{n+1}-w\right\|^{2}= & \left\|\left(1-\delta_{n}\right) x_{n}+\delta_{n} \vartheta_{n}-w\right\|^{2} \\
= & \left\|\left(1-\delta_{n}\right)\left(x_{n}-w\right)+\delta_{n}\left(\vartheta_{n}-w\right)\right\|^{2} \\
= & \left(1-\delta_{n}\right)\left\|x_{n}-w\right\|^{2}+\delta_{n}\left\|\vartheta_{n}-w\right\|^{2} \\
& -\delta_{n}\left(1-\delta_{n}\right)\left\|x_{n}-\vartheta_{n}\right\|^{2} .
\end{aligned}
$$

From $z_{n}=\left(1-\tau_{n}\right) x_{n}+\tau_{n} \zeta_{n}$ and $x_{n+1}=\left(1-\delta_{n}\right) x_{n}+\delta_{n} \vartheta_{n}$, we obtain

$$
\zeta_{n}-x_{n}=\frac{1}{\tau_{n}}\left(z_{n}-x_{n}\right)
$$

and

$$
\vartheta_{n}-x_{n}=\frac{1}{\delta_{n}}\left(x_{n+1}-x_{n}\right) .
$$


Using (3.12), (3.13), and (3.14), we obtain

$$
\begin{aligned}
\left\|x_{n+1}-w\right\|^{2} \leq & \left(1-\delta_{n}\right)\left\|x_{n}-w\right\|^{2}+\delta_{n}\left\|\vartheta_{n}-w\right\|^{2} \\
& -\delta_{n}\left(1-\delta_{n}\right)\left\|x_{n}-\vartheta_{n}\right\|^{2} \\
= & \left(1-\delta_{n}\right)\left\|x_{n}-w\right\|^{2}+\delta_{n}\left\|z_{n}-w\right\|^{2} \\
& -\frac{\left(1-\delta_{n}\right)}{\delta_{n}}\left\|x_{n+1}-x_{n}\right\|^{2} .
\end{aligned}
$$

From (3.14), we get

$$
\begin{aligned}
\left\|z_{n}-w\right\|^{2}= & \left\|\left(1-\tau_{n}\right) x_{n}+\tau_{n} \zeta_{n}-w\right\|^{2} \\
= & \left\|\left(1-\tau_{n}\right)\left(x_{n}-w\right)+\tau_{n}\left(\zeta_{n}-w\right)\right\|^{2} \\
= & \left(1-\tau_{n}\right)\left\|x_{n}-w\right\|^{2}+\tau_{n}\left\|\zeta_{n}-w\right\|^{2} \\
& -\tau_{n}\left(1-\tau_{n}\right)\left\|x_{n}-\zeta_{n}\right\|^{2} \\
= & \left(1-\tau_{n}\right)\left\|x_{n}-w\right\|^{2}+\tau_{n}\left\|\zeta_{n}-w\right\|^{2} \\
& -\frac{\left(1-\tau_{n}\right)}{\tau_{n}}\left\|z_{n}-x_{n}\right\|^{2} .
\end{aligned}
$$

From (3.9), we get

$$
\begin{aligned}
\left\|z_{n}-w\right\|^{2} \leq & \left(1-\tau_{n}\right)\left\|x_{n}-w\right\|^{2}+\tau_{n}\left\|w_{n}-w\right\|^{2} \\
& -\frac{\left(1-\tau_{n}\right)}{\tau_{n}}\left\|z_{n}-x_{n}\right\|^{2} .
\end{aligned}
$$

Using (3.15), (3.16), and (3.17), we obtain

$$
\begin{aligned}
\left\|x_{n+1}-w\right\|^{2} \leq & \left(1-\delta_{n}\right)\left\|x_{n}-w\right\|^{2}+\delta_{n}\left(1-\tau_{n}\right)\left\|x_{n}-w\right\|^{2}+\delta_{n} \tau_{n}\left\|w_{n}-w\right\|^{2} \\
& -\frac{\delta_{n}\left(1-\tau_{n}\right)}{\tau_{n}}\left\|z_{n}-x_{n}\right\|^{2} \\
& -\frac{\left(1-\delta_{n}\right)}{\delta_{n}}\left\|x_{n+1}-x_{n}\right\|^{2} \\
= & \left(1-\delta_{n} \tau_{n}\right)\left\|x_{n}-w\right\|^{2}+\delta_{n} \tau_{n}\left\|w_{n}-w\right\|^{2} \\
& -\frac{\delta_{n}\left(1-\tau_{n}\right)}{\tau_{n}}\left\|z_{n}-x_{n}\right\|^{2} \\
& -\frac{\left(1-\delta_{n}\right)}{\delta_{n}}\left\|x_{n+1}-x_{n}\right\|^{2}
\end{aligned}
$$

and

$$
\begin{aligned}
\left\|w_{n}-w\right\|^{2}= & \left\|x_{n}+\theta_{n}\left(x_{n}-x_{n-1}\right)-w\right\|^{2} \\
= & \left\|\left(1+\theta_{n}\right)\left(x_{n}-w\right)-\theta_{n}\left(x_{n-1}-w\right)\right\|^{2} \\
= & \left(1+\theta_{n}\right)\left\|x_{n}-w\right\|^{2}-\theta_{n}\left\|x_{n-1}-w\right\|^{2} \\
& +\theta_{n}\left(1+\theta_{n}\right)\left\|x_{n}-x_{n-1}\right\|^{2} .
\end{aligned}
$$


Using (3.18) and (3.19), we obtain

$$
\begin{aligned}
\left\|x_{n+1}-w\right\|^{2} \leq & \left(1-\delta_{n} \tau_{n}\right)\left\|x_{n}-w\right\|^{2}+\delta_{n} \tau_{n}\left(1+\theta_{n}\right)\left\|x_{n}-w\right\|^{2} \\
& -\delta_{n} \tau_{n} \theta_{n}\left\|x_{n-1}-w\right\|^{2}+\delta_{n} \tau_{n} \theta_{n}\left(1+\theta_{n}\right)\left\|x_{n}-x_{n-1}\right\|^{2} \\
& -\frac{\delta_{n}\left(1-\tau_{n}\right)}{\tau_{n}}\left\|z_{n}-x_{n}\right\|^{2} \\
& -\frac{\left(1-\delta_{n}\right)}{\delta_{n}}\left\|x_{n+1}-x_{n}\right\|^{2} \\
= & \left(1+\delta_{n} \tau_{n} \theta_{n}\right)\left\|x_{n}-w\right\|^{2} \\
& -\delta_{n} \tau_{n} \theta_{n}\left\|x_{n-1}-w\right\|^{2}+\delta_{n} \tau_{n} \theta_{n}\left(1+\theta_{n}\right)\left\|x_{n}-x_{n-1}\right\|^{2} \\
& -\frac{\delta_{n}\left(1-\tau_{n}\right)}{\tau_{n}}\left\|z_{n}-x_{n}\right\|^{2} \\
& -\frac{\left(1-\delta_{n}\right)}{\delta_{n}}\left\|x_{n+1}-x_{n}\right\|^{2} .
\end{aligned}
$$

Let $\Upsilon_{n}=\left\|x_{n}-w\right\|^{2}-\delta_{n} \tau_{n} \theta_{n}\left\|x_{n-1}-w\right\|^{2}+\delta_{n} \tau_{n} \theta_{n}\left(1+\theta_{n}\right)\left\|x_{n}-x_{n-1}\right\|^{2}$.

Since $\delta_{n} \leq \delta_{n+1}, \quad \tau_{n} \leq \tau_{n+1}$ and $\theta_{n} \leq \theta_{n+1}$ then $\delta_{n} \tau_{n} \theta_{n} \leq \delta_{n+1} \tau_{n+1} \theta_{n+1}$. Hence,

$$
\begin{aligned}
\Upsilon_{n+1}-\Upsilon_{n} \leq & \left\|x_{n+1}-w\right\|^{2}-\left(1+\delta_{n+1} \tau_{n+1} \theta_{n+1}\right)\left\|x_{n}-w\right\|^{2} \\
& +\delta_{n} \tau_{n} \theta_{n}\left\|x_{n-1}-w\right\|^{2}+\delta_{n+1} \tau_{n+1} \theta_{n+1}\left(1+\theta_{n+1}\right)\left\|x_{n+1}-x_{n}\right\|^{2} \\
& -\delta_{n} \tau_{n} \theta_{n}\left(1+\theta_{n}\right)\left\|x_{n}-x_{n-1}\right\|^{2} \\
\leq & \left\|x_{n+1}-w\right\|^{2}-\left(1+\delta_{n} \tau_{n} \theta_{n}\right)\left\|x_{n}-w\right\|^{2} \\
& +\delta_{n} \tau_{n} \theta_{n}\left\|x_{n-1}-w\right\|^{2}+\delta_{n+1} \tau_{n+1} \theta_{n+1}\left(1+\theta_{n+1}\right)\left\|x_{n+1}-x_{n}\right\|^{2} \\
& -\delta_{n} \tau_{n} \theta_{n}\left(1+\theta_{n}\right)\left\|x_{n}-x_{n-1}\right\|^{2} .
\end{aligned}
$$

Using (3.20) and (3.21), we obtain

$$
\begin{aligned}
\Upsilon_{n+1}-\Upsilon_{n} & \leq-\frac{\left(1-\delta_{n}\right)}{\delta_{n}}\left\|x_{n+1}-x_{n}\right\|^{2}+\delta_{n+1} \tau_{n+1} \theta_{n+1}\left(1+\theta_{n+1}\right)\left\|x_{n+1}-x_{n}\right\|^{2} \\
& =-\left(\frac{1-\delta_{n}}{\delta_{n}}-\delta_{n+1} \tau_{n+1} \theta_{n+1}\left(1+\theta_{n+1}\right)\right)\left\|x_{n+1}-x_{n}\right\|^{2} .
\end{aligned}
$$

Using conditions (i), (ii), and (iv), we obtain

$$
\begin{aligned}
\frac{1-\delta_{n}}{\delta_{n}}-\delta_{n+1} \tau_{n+1} \theta_{n+1}\left(1+\theta_{n+1}\right) & =\frac{1}{\delta_{n}}-1-\delta_{n+1} \tau_{n+1} \theta_{n+1}\left(1+\theta_{n+1}\right) \\
& \geq 2+\beta-1-\frac{2}{(2+\beta)(2+\alpha)} \\
& \geq \beta .
\end{aligned}
$$

Using (3.22) and (3.23), we obtain

$$
\Upsilon_{n+1}-\Upsilon_{n} \leq-\beta\left\|x_{n+1}-x_{n}\right\|^{2} .
$$

Hence, the sequence $\left\{\Upsilon_{n}\right\}$ is non-increasing. Similarly,

$$
\begin{aligned}
\Upsilon_{n} & =\left\|x_{n}-w\right\|^{2}-\delta_{n} \tau_{n} \theta_{n}\left\|x_{n-1}-w\right\|^{2}+\delta_{n} \tau_{n} \theta_{n}\left(1+\theta_{n}\right)\left\|x_{n}-x_{n-1}\right\|^{2} \\
& \geq \mid x_{n}-w\left\|^{2}-\delta_{n} \tau_{n} \theta_{n}\right\| x_{n-1}-w \|^{2}
\end{aligned}
$$

where

$$
\delta_{n} \tau_{n} \theta_{n} \leq \frac{1}{(2+\beta)(2+\alpha)}=\varepsilon \epsilon<1 .
$$


From (3.24), we get

$$
\begin{aligned}
\left\|x_{n}-w\right\|^{2} & \leq \delta_{n} \tau_{n} \theta_{n}\left\|x_{n-1}-w\right\|^{2}+\Upsilon_{n} \\
& \leq \varepsilon \epsilon\left\|x_{n-1}-w\right\|^{2}+\Upsilon_{n} \\
& \leq \varepsilon \epsilon\left\|x_{n-1}-w\right\|^{2}+\Upsilon_{1}
\end{aligned}
$$

and

$$
\begin{aligned}
\Upsilon_{n+1}= & \left\|x_{n+1}-w\right\|^{2}-\delta_{n+1} \tau_{n+1} \theta_{n+1}\left\|x_{n}-w\right\|^{2} \\
& +\delta_{n+1} \tau_{n+1} \theta_{n+1}\left(1+\theta_{n+1}\right)\left\|x_{n+1}-x_{n}\right\|^{2} \\
\geq & \mid x_{n}-w\left\|^{2}-\delta_{n} \tau_{n} \theta_{n}\right\| x_{n-1}-w \|^{2} .
\end{aligned}
$$

From (3.27) and (3.26), we get

$$
\begin{aligned}
-\Upsilon_{n+1} & \leq \delta_{n+1} \tau_{n+1} \theta_{n+1}\left\|x_{n}-w\right\|^{2} \\
& \leq \varepsilon \epsilon\left\|x_{n}-w\right\|^{2}
\end{aligned}
$$

$$
\leq(\varepsilon \epsilon)^{n+1}\left\|x_{0}-w\right\|^{2}+\frac{\varepsilon \epsilon \Upsilon_{1}}{1-\varepsilon \epsilon}
$$

Using (3.24) and (3.28), we obtain

$$
\begin{aligned}
\beta \sum_{n=1}^{k}\left\|x_{n+1}-x_{n}\right\|^{2} & \leq \Upsilon_{1}-\Upsilon_{k+1} \\
& \leq(\varepsilon \epsilon)^{k+1}\left\|x_{0}-w\right\|^{2}+\frac{\Upsilon_{1}}{1-\varepsilon \epsilon} .
\end{aligned}
$$

This implies

$$
\sum_{n=1}^{\infty}\left\|x_{n+1}-x_{n}\right\|^{2} \leq \frac{\Upsilon_{1}}{\beta(1-\varepsilon \epsilon)}<\infty .
$$

Hence, $\lim _{n \rightarrow \infty}\left\|x_{n+1}-x_{n}\right\|^{2}=0$, we get

$$
\begin{aligned}
\left\|w_{n}-x_{n}\right\|^{2} & =\theta_{n}\left\|x_{n}-x_{n-1}\right\|^{2} \\
& \leq\left\|x_{n}-x_{n-1}\right\|^{2} \rightarrow 0 \text { as } n \rightarrow \infty .
\end{aligned}
$$

From (3.20), we get

$$
\left\|x_{n+1}-w\right\|^{2} \leq\left(1+\delta_{n} \tau_{n} \theta_{n}\right)\left\|x_{n}-w\right\|^{2}-\delta_{n} \tau_{n} \theta_{n}\left\|x_{n-1}-w\right\|^{2}+2\left\|x_{n}-x_{n-1}\right\|^{2} .
$$

Using Lemma 2.4, we obtain

$$
\lim _{n \rightarrow \infty}\left\|x_{n}-w\right\|^{2}=q<\infty .
$$

Using condition (i), we get $\lim _{n \rightarrow \infty} \theta_{n}$ exists. Suppose $\lim _{n \rightarrow \infty} \theta_{n}=\theta \in[0,1]$ then $\lim _{n \rightarrow \infty} \theta_{n}\left\|x_{n}-w\right\|^{2}=\theta q$. Similarly, $\lim _{n \rightarrow \infty} \theta_{n}\left\|x_{n-1}-w\right\|^{2}=\theta q$ and from (3.19), we get

$$
\begin{aligned}
\lim _{n \rightarrow \infty}\left\|w_{n}-w\right\|^{2}= & \lim _{n \rightarrow \infty}\left[\left(1+\theta_{n}\right)\left\|x_{n}-w\right\|^{2}-\theta_{n}\left\|x_{n-1}-w\right\|^{2}\right. \\
& \left.+\theta_{n}\left(1+\theta_{n}\right)\left\|x_{n}-x_{n-1}\right\|^{2}\right]=q .
\end{aligned}
$$


Hence, $\lim _{n \rightarrow \infty}\left[\left(1+\theta_{n}\right)\left\|x_{n}-w\right\|^{2}-\theta_{n}\left\|x_{n-1}-w\right\|^{2}+\theta_{n}\left(1+\theta_{n}\right)\left\|x_{n}-x_{n-1}\right\|^{2}\right]$ exists. Using (3.8) and (3.18), we obtain

$$
\begin{aligned}
\left\|x_{n+1}-w\right\|^{2} \leq & \left(1-\delta_{n} \tau_{n}\right)\left\|x_{n}-w\right\|^{2}+\delta_{n} \tau_{n}\left\|w_{n}-w\right\|^{2} \\
& -\delta_{n} \tau_{n} \gamma_{n}\left\|T_{n} w_{n}-w_{n}\right\|^{2} .
\end{aligned}
$$

So, we get

$$
\begin{aligned}
\delta \tau \gamma\left\|T_{n} w_{n}-w_{n}\right\|^{2} \leq & \delta_{n} \tau_{n} \gamma_{n}\left\|T_{n} w_{n}-w_{n}\right\|^{2} \\
\leq & \left\|x_{n}-w\right\|^{2}-\left\|x_{n+1}-w\right\|^{2}-\delta_{n} \tau_{n}\left\|x_{n}-w\right\|^{2} \\
& +\delta_{n} \tau_{n}\left\|w_{n}-w\right\|^{2} .
\end{aligned}
$$

By condition (ii) and (iv), we get $\lim _{n \rightarrow \infty} \delta_{n}$ and $\lim _{n \rightarrow \infty} \tau_{n}$ exists. From

$$
\lim _{n \rightarrow \infty} \delta_{n} \tau_{n}\left\|x_{n}-w\right\|^{2}=\lim _{n \rightarrow \infty} \delta_{n} \tau_{n}\left\|w_{n}-w\right\|^{2}
$$

and (3.36), we get $\lim \sup _{n \rightarrow \infty}\left\|T_{n} w_{n}-w_{n}\right\|^{2} \leq 0$. Then,

$$
\lim _{n \rightarrow \infty}\left\|T_{n} w_{n}-w_{n}\right\|=0 \text {. }
$$

Hence,

$$
\begin{aligned}
\left\|x_{n}-T_{n} x_{n}\right\| & \leq\left\|x_{n}-w_{n}\right\|+\left\|w_{n}-T_{n} w_{n}\right\|+\left\|T_{n} w_{n}-T_{n} x_{n}\right\| \\
& \leq 2\left\|x_{n}-w_{n}\right\|+\left\|w_{n}-T_{n} w_{n}\right\| \rightarrow 0 \text { as } n \rightarrow \infty .
\end{aligned}
$$

Since $\left\{T_{n}\right\}$ satisfies NST*-condition, we get $\omega_{w}\left(x_{n}\right) \subset \Omega:=\bigcap_{n=1}^{\infty}$ Fix $\left(T_{n}\right)$. Hence, by Lemma 2.2, we obtain that $\left\{x_{n}\right\}$ converges weakly to a point $w \in \Omega$.

Finally, we apply Algorithm (3.7) for solving the minimization problem (1.3) by setting $T_{n}=\operatorname{prox}_{r_{n} g}\left(I-r_{n} \nabla f\right)$, the forward-backward operator of $f$ and $g$ with respect to $r_{n}$, where $g: \mathcal{H} \rightarrow \mathbb{R} \cup\{\infty\}$ is proper convex and lower semi-continuous, and $f: \mathcal{H} \rightarrow \mathbb{R}$ is a continuously differentiable convex function, whose gradient is Lipschitz continuous $(L>0)$.

Theorem 3.2. Let $\mathcal{H}$ be a Hilbert space, $f: \mathcal{H} \rightarrow \mathbb{R}$ be a convex and differentiable function such that $\nabla f$ is $\frac{1}{L}$-Lipschitz continuous and let $g: \mathcal{H} \rightarrow \mathbb{R} \cup\{\infty\}$ be a proper convex and lower semi-continuous function. We define a sequence $\left\{x_{n}\right\}$ by the iterative scheme, for any $x_{0}, x_{1} \in \mathcal{H}$,

$$
\left\{\begin{array}{l}
w_{n}=x_{n}+\theta_{n}\left(x_{n}-x_{n-1}\right), \\
z_{n}=\left(1-\tau_{n}\right) x_{n}+\tau_{n}\left(\left(1-\gamma_{n}\right) w_{n}+\gamma_{n} \operatorname{prox}_{r_{n} g}\left(w_{n}-r_{n} \nabla f\left(w_{n}\right)\right)\right), \\
x_{n+1}=\left(1-\delta_{n}\right) x_{n}+\delta_{n}\left(\left(1-\lambda_{n}\right) z_{n}+\lambda_{n} \operatorname{prox}_{r_{n} g}\left(z_{n}-r_{n} \nabla f\left(z_{n}\right)\right)\right),
\end{array}\right.
$$

where $\left\{\tau_{n}\right\},\left\{\gamma_{n}\right\},\left\{\delta_{n}\right\},\left\{\lambda_{n}\right\},\left\{r_{n}\right\}$ and $\left\{\theta_{n}\right\}$ are sequences such that

(i) $0 \leq \theta_{n} \leq \theta_{n+1} \leq 1$;

(ii) $0<\tau \leq \tau_{n} \leq \tau_{n+1} \leq \frac{1}{2+\alpha}:=\epsilon, \alpha>0$;

(iii) $0<\gamma \leq \gamma_{n} \leq \rho<1$;

(iv) $0<\delta \leq \delta_{n} \leq \delta_{n+1} \leq \frac{1}{2+\beta}:=\varepsilon, \beta>0$;

(v) $0<\lambda \leq \lambda_{n} \leq \iota<1$;

(vi) $0<r_{n}<\frac{2}{L}$.

Then the sequence $\left\{x_{n}\right\}$ generated by (3.37) converges weakly to a point $w \in \arg \min (f+g)$.

Proof. Let $T$ be the forward-backward operator of $f$ and $g$ with respect to $r$, and $T_{n}$ be the forward-backward operator of $f$ and $g$ with respect to $r_{n}$, that is $T:=\operatorname{prox}_{r g}(I-r \nabla f)$ and $T_{n}:=\operatorname{prox}_{r_{n} g}\left(I-r_{n} \nabla f\right)$. Then $T$ and $\left\{T_{n}\right\}$ are nonexpansive operators for all $n$, 
and Fix $(T)=\bigcap_{n=1}^{\infty}$ Fix $\left(T_{n}\right)=\arg \min (f+h)$ (see Proposition 26.1 in [1].) Using Lemma 2.3 , we have that $\left\{T_{n}\right\}$ satisfies the NST*-condition. Hence, we obtain the required result directly by Theorem 3.1.

\section{NUMERICAL EXPERIMENTS}

In this section, we will discuss the results of some of the tests using our proposed algorithm in Theorem 3.2 to solve the problem of image restoration. In Theorem 3.2, we set $f(x)=\frac{1}{2}\|A x-h\|_{2}^{2}$ and $g(x)=\mu\|x\|_{1}$, it is easy to see that $f$ is a smooth function with $L$-Lipschitz continuous gradient $\nabla f(x)=A^{*}(A x-h)$, where $L=\left\|A^{*} A\right\|$. The 1-norm is "simple", as its proximal operator is a soft thresholding: $\operatorname{prox}_{r g}\left(x_{n}\right)=\max \left(0,1-\frac{\mu r}{\left|x_{n}\right|}\right) x_{n}$. We consider two blurring functions from MATLAB: a Gaussian blur (Matlab function is, "fspecial('gaussian' $, 7,7$ )") and a motion blur (Matlab function is, "fspecial('motion',15,45)") respectively.

Numerical experiments presented in this section aim at proving the validity of our proposed algorithm compared with NAGA proposed in (1.6). We set $\theta_{n}=0.99, \mu=$ $10^{-4}, \tau_{n}=\gamma_{n}=\delta_{n}=\lambda_{n}=0.91$. In our paper, the comparison is done in terms of the relative error defined as

$$
\frac{\left\|x_{n}-x\right\|_{2}^{2}}{\|x\|_{2}^{2}}
$$

the quality of image recovery is measured by the improvement in signal to noise ratio (ISNR). Note that ISNR defined as

$$
\text { ISNR }=10 \log \frac{\|x-h\|_{2}^{2}}{\left\|x-x_{n}\right\|_{2}^{2}},
$$

where $x, h$, and $x_{n}$ are the original image, the observed image, and estimated image at iteration $n$, respectively. All algorithms are implemented under Windows 10 and MATLAB 2017b running on a Dell laptop with Intel(R) Core(TM) i5 CPU and 4 GB of RAM. The stopping criterion of the algorithm is

$$
\frac{\left\|x_{n+1}-x_{n}\right\|_{2}}{\left\|x_{n+1}\right\|_{2}}<10^{-4}
$$

The test images are Kitkuan $(256 \times 256)$, Butterfly $(256 \times 256)$, HeadCT $(256 \times 256)$ and Brain $(256 \times 256)$, which show in Figure 3 .

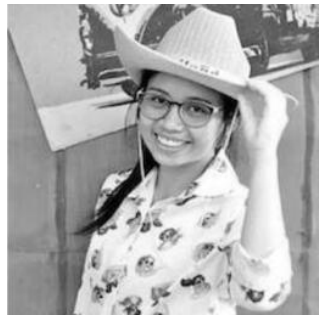

(A) Kitkuan

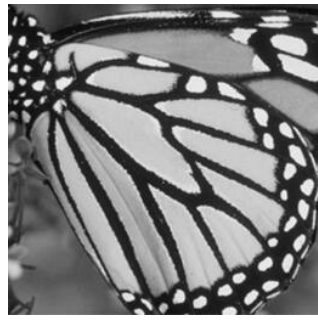

(B) Butterfly

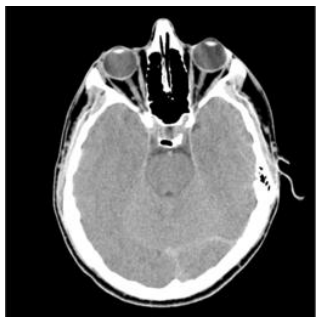

(C) HeadCT

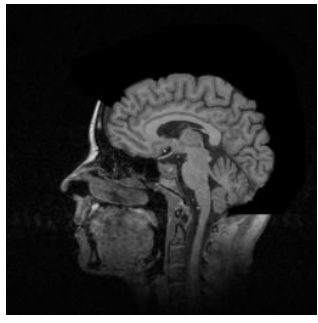

(D) Brain

FIGURE 3. Test images

We show numerical results for case Gaussian blur in Table 1, restoration image results for case Gaussian blur in Figure 4, ISNR and relative error results for case Gaussian blur 
in Figure 5, numerical results for case Motion blur in Table 2, restoration image results for case Motion blur in Figure 6, ISNR results for case Motion blur in Figure 7

TABLE 1. Numerical results in case Gaussian blur

\begin{tabular}{|c|c|c|c|c|c|c|c|c|}
\hline \multirow{2}{*}{ Figure } & \multicolumn{4}{|c|}{ Our algorithm } & \multicolumn{4}{c|}{ NAGA } \\
\cline { 2 - 9 } & Iter. & Time & ISNR & Relative error & Iter & Time & ISNR & Relative error \\
\hline Kitkuan & 149 & 1.73 & 6.14 & $2.49 \times 10^{-3}$ & 362 & 3.79 & 4.70 & $3.48 \times 10^{-3}$ \\
\hline Butterfly & 268 & 4.29 & 7.74 & $5.68 \times 10^{-3}$ & 733 & 10.66 & 6.82 & $7.03 \times 10^{-3}$ \\
\hline HeadCT & 277 & 2.38 & 9.10 & $2.82 \times 10^{-3}$ & 893 & 7.68 & 7.54 & $4.05 \times 10^{-3}$ \\
\hline Brain & 264 & 2.99 & 6.51 & $5.71 \times 10^{-3}$ & 753 & 7.90 & 5.71 & $7.08 \times 10^{-3}$ \\
\hline
\end{tabular}

TABLE 2. Numerical results in case Motion blur

\begin{tabular}{|c|c|c|c|c|c|c|c|c|}
\hline \multirow{2}{*}{ Figure } & \multicolumn{4}{|c|}{ Our algorithm } & \multicolumn{4}{c|}{ NAGA } \\
\cline { 2 - 9 } & Iter. & Time & ISNR & Relative error & Iter & Time & ISNR & Relative error \\
\hline Kitkuan & 157 & 1.51 & 11.51 & $1.63 \times 10^{-3}$ & 606 & 5.48 & 10.04 & $2.09 \times 10^{-3}$ \\
\hline Butterfly & 321 & 3.15 & 14.22 & $4.42 \times 10^{-3}$ & 1155 & 10.51 & 13.89 & $4.77 \times 10^{-3}$ \\
\hline HeadCT & 277 & 2.52 & 18.99 & $7.29 \times 10^{-4}$ & 1088 & 23.65 & 17.69 & $9.85 \times 10^{-4}$ \\
\hline Brain & 288 & 2.80 & 16.01 & $1.85 \times 10^{-3}$ & 1023 & 8.56 & 15.55 & $2.05 \times 10^{-3}$ \\
\hline
\end{tabular}

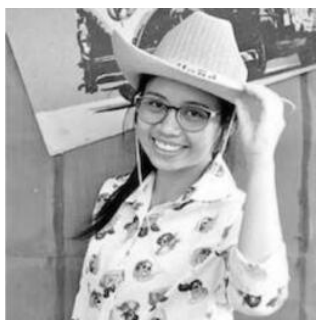

(A) Original

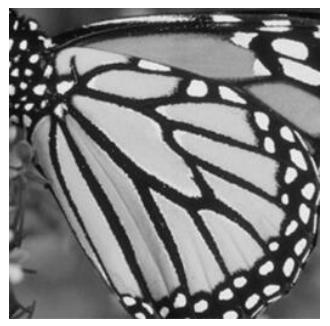

(E) Original

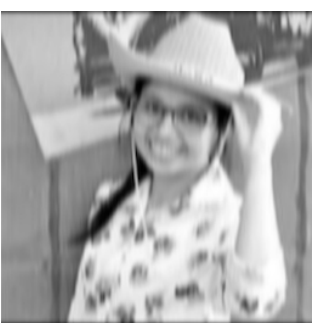

(B) Observed

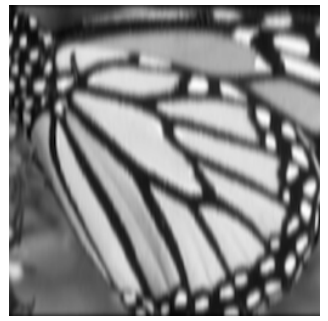

(F) Observed

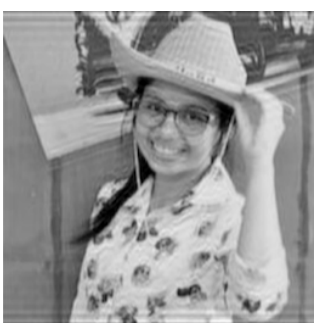

(C) Our

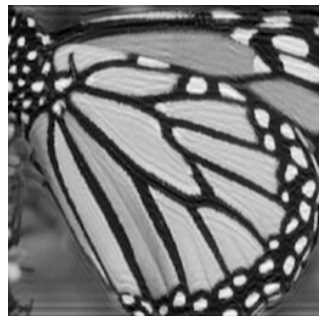

(G) Our

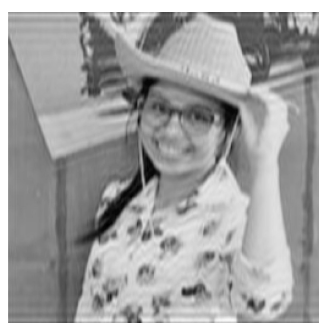

(D) NAGA

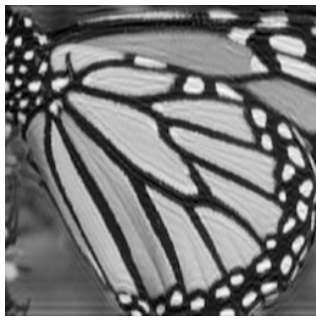

(H) NAGA 


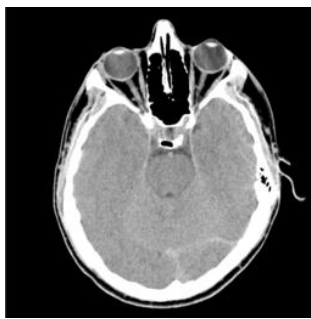

(A) Original

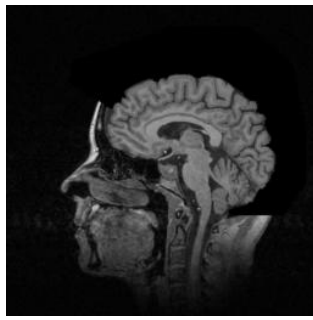

(E) Original

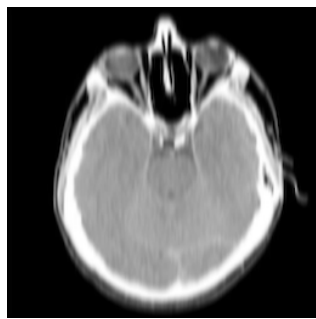

(B) Observed

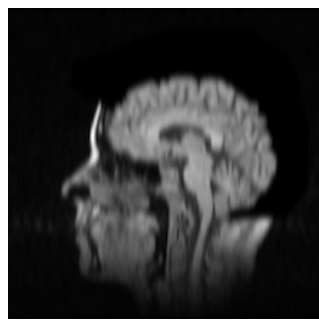

(F) Observed

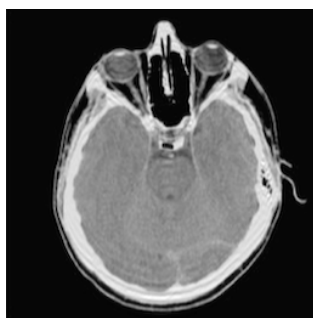

(c) Our

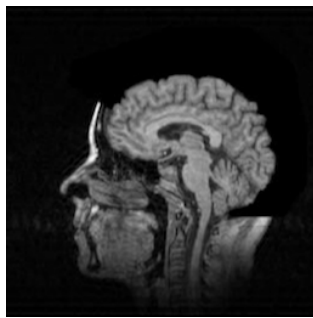

(G) Our

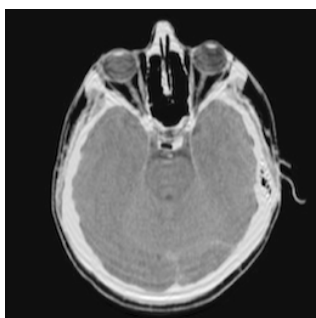

(D) NAGA

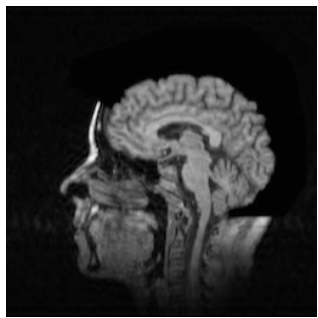

(H) NAGA

FIGURE 4. Restoration image results in case Gaussian blur

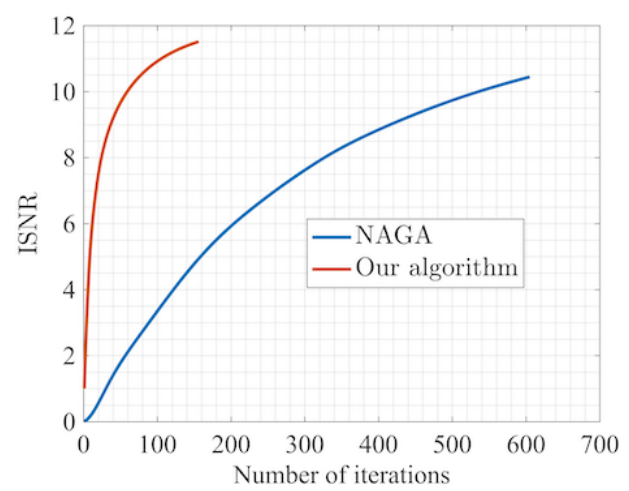

(A) Kitkuan ISNR results (Gaussian)

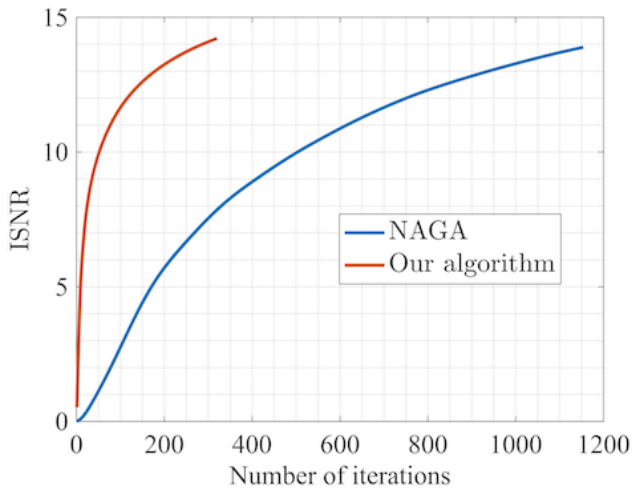

(C) Butterfly ISNR results (Gaussian)

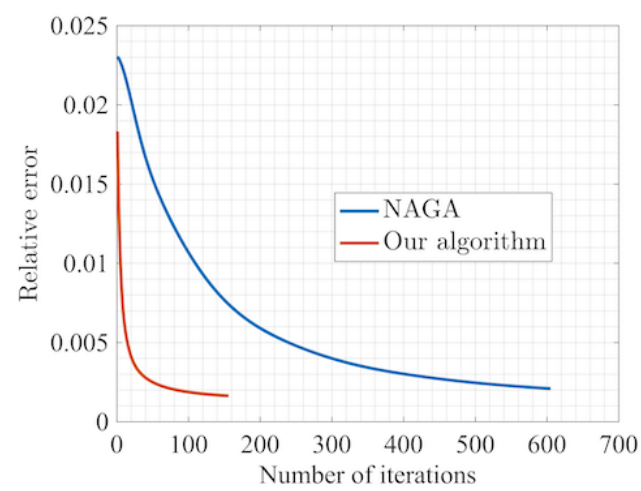

(B) Kitkuan relative error results (Gaussian)

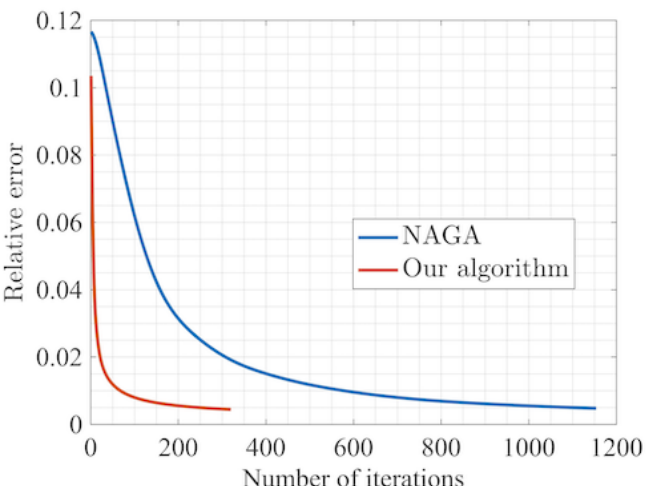

(D) Butterfly relative error results (Gaussian) 


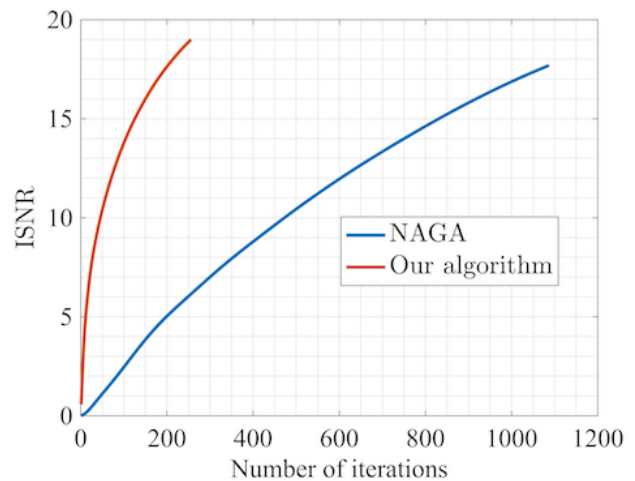

(A) HeadCT ISNR results (Gaussian)

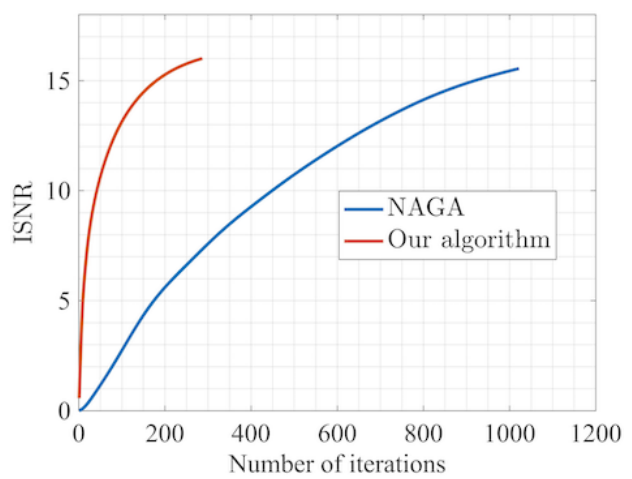

(C) Brain ISNR results (Gaussian)

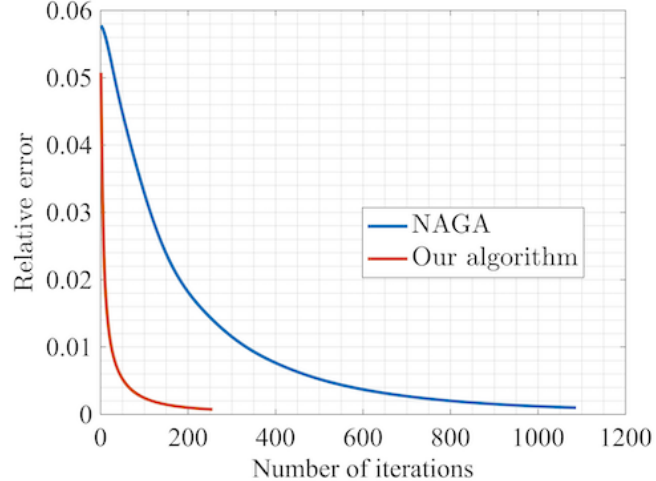

(в) HeadCT relative error results (Gaussian)

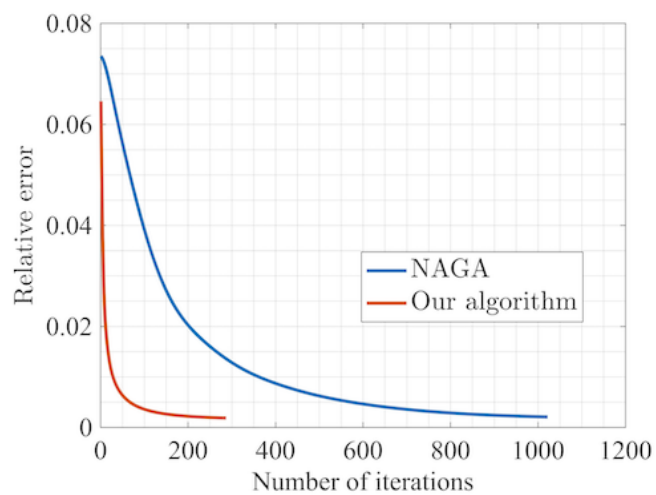

(D) Brain relative error results (Gaussian)

FIGURE 5. ISNR and relative error (Gaussian)

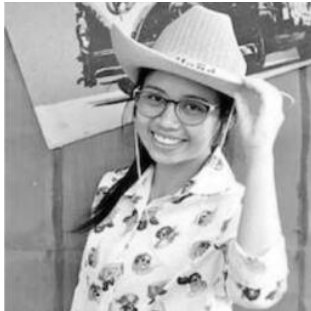

(A) Original

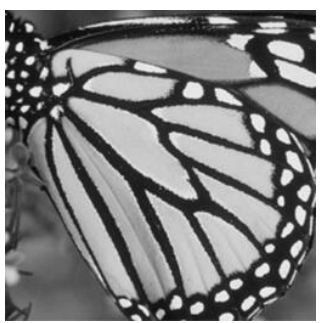

(E) Original

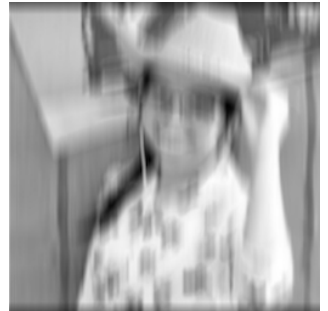

(B) Observed

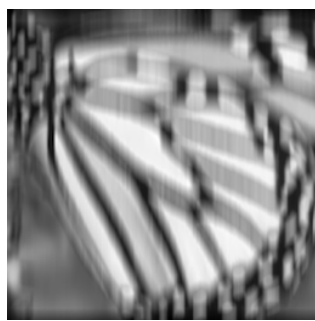

(F) Observed

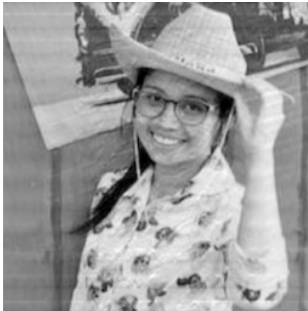

(C) Our

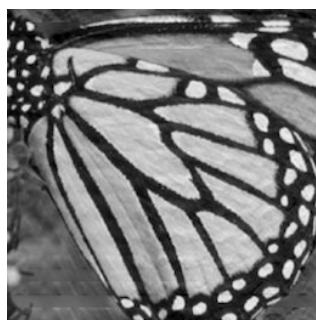

(G) Our

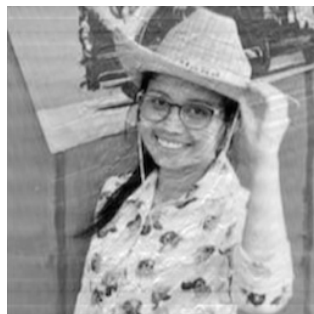

(D) NAGA

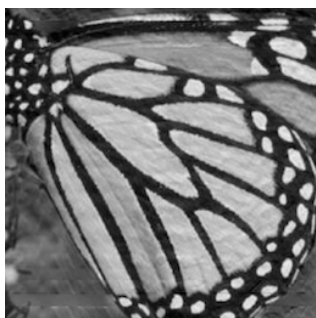

(H) NAGA 


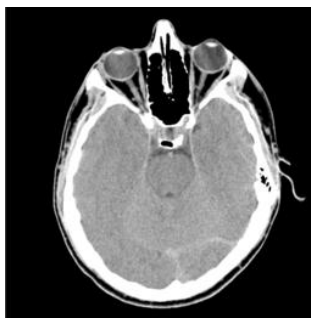

(A) Original

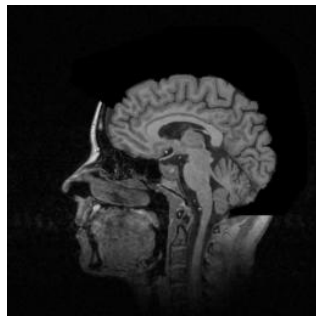

(E) Original

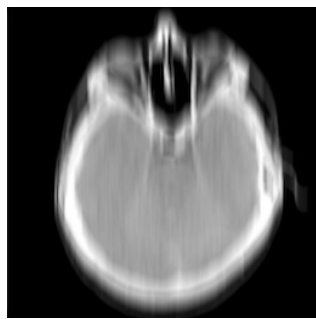

(B) Observed

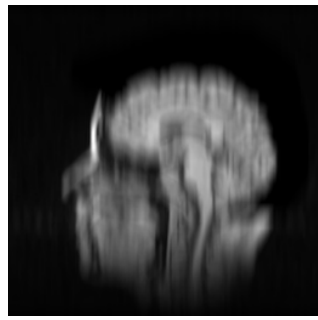

(F) Observed

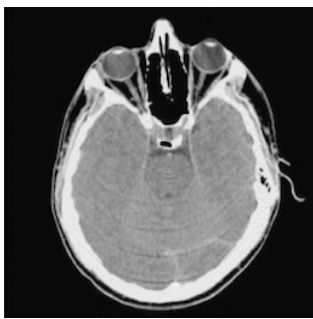

(c) Our

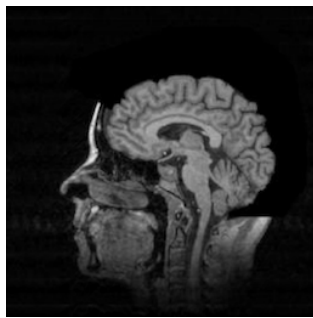

(G) Our

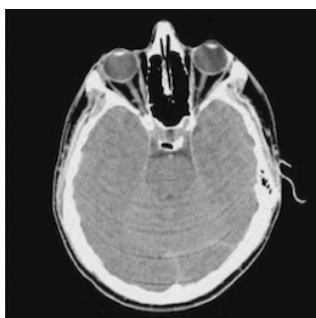

(D) NAGA

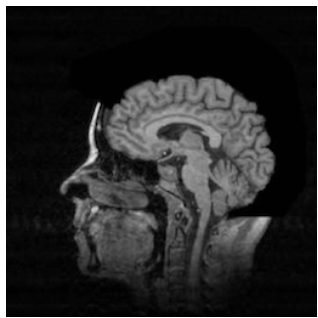

(H) NAGA

FIGURE 6. Restoration image result in case Motion blur

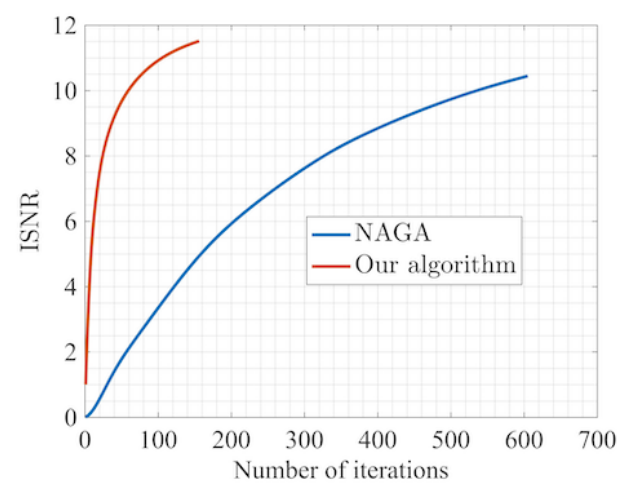

(A) Kitkuan ISNR results (Motion)

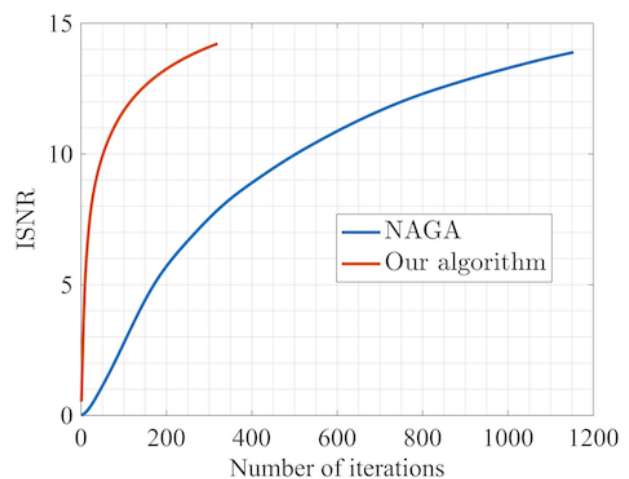

(C) Butterfly ISNR results (Motion)

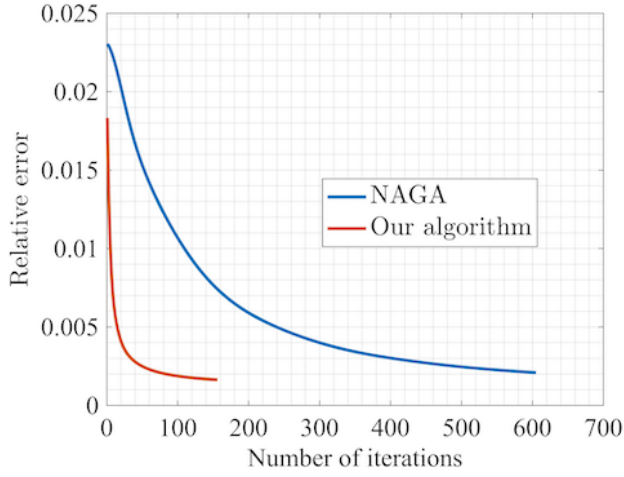

(B) Kitkuan relative error results (Motion)

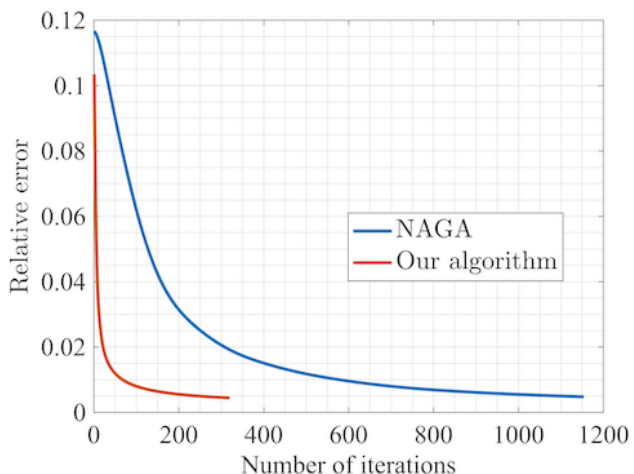

(D) Butterfly relative error results (Motion) 


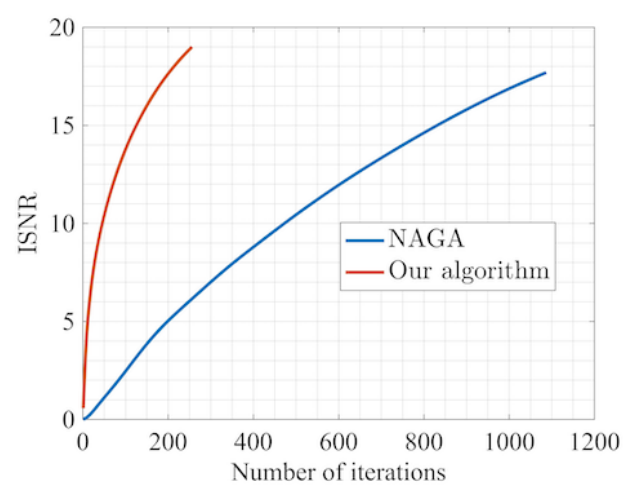

(A) HeadCT ISNR results (Motion)

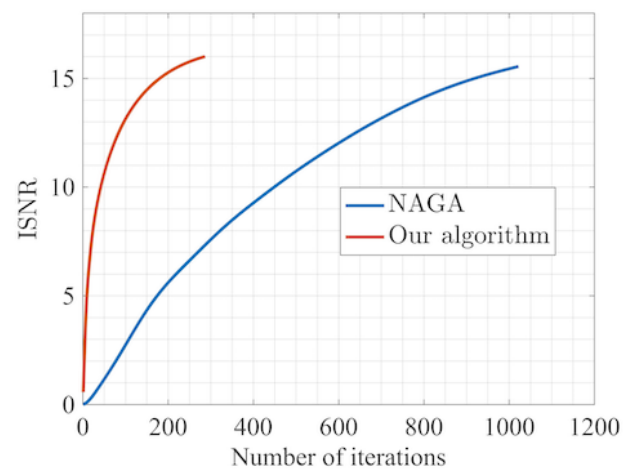

(C) Brain ISNR results (Motion)

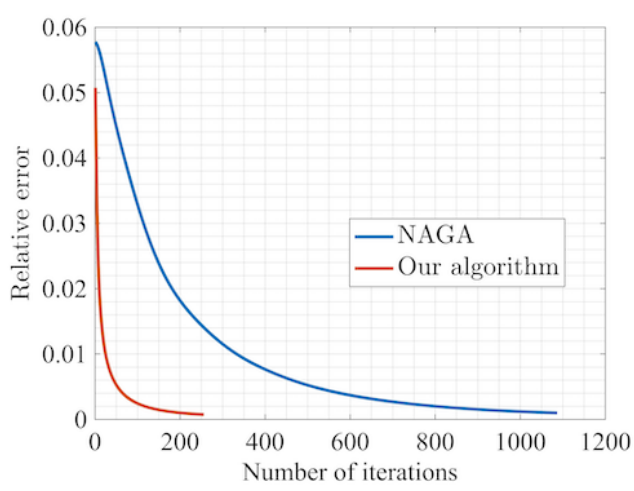

(в) HeadCT relative error results (Motion)

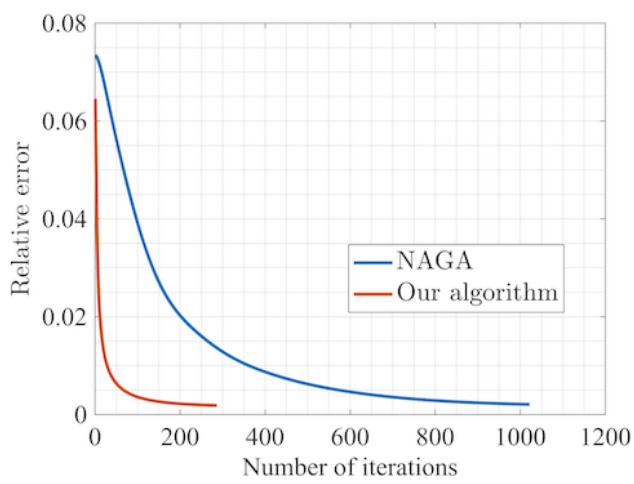

(D) Brain relative error results (Motion)

FIGURE 7. ISNR and relative error (Motion)

\section{CONCLUSION}

In this paper, we present the iterative methods using the ideas of the Ishikawa type and inertial technique to solving optimization problems and image restoration problems. A convergence theorem of our proposed methods, Theorem 3.1, is established and proved under some suitable conditions. We applied our main result to solving a minimization problem in the form of the sum of two proper lower semi-continuous and convex functions. As applications, we applied our algorithm (3.37), to solving image restoration problems. Moreover, we did some numerical experiments to illustrate the performance of the studied algorithms and show that ISNR of algorithm (3.37) is better than those of the NAGA [22].

Acknowledgement. This project was supported by the Research and Development Institute, Rambhai Barni Rajabhat University (Grant no.2240/2563).

\section{REFERENCES}

[1] Bauschke, H. H.; Combettes, P. L. Convex Analysis and Monotone Operator Theory in Hilbert Spaces, Springer, New York, 2011.

[2] Beck, A.; Teboulle, M. A fast iterative shrinkage-thresholding algorithm for linear inverse problems. SIAM Journal on Imaging Sciences 2 (2009), no. 1 183-202. 
[3] Burachik, R. S.; Iusem, A. N. Set-valued mappings and enlargements of monotone operator. 8, Springer Science Business Media, New York, 2007.

[4] Bussaban, L.; Suantai, S.; Kaewkhao, A. A parallel inertial S-iteration forward-backward algorithm for regression and classification problems, Carpathian J. Math., 36 (2020), 21-30.

[5] Combette, P. L.; Dung, D.; Vu, B. C. Dualization of signal recovery problems. Set-Valued Analysis 18 (2010), 373-404.

[6] Goebel, K.; Reich, S. Uniform convexity, hyperbolic geometry, and nonexpansive mappings, Marcel Dekker, New York, 1984.

[7] Hanjing, A.; Suantai, S. A fast image restoration algorithm based on a fixed point and optimization method. Mathematics 8 (2020), 378.

[8] Kellogg, R. B. Nonlinear alternating direction algorithm. Math. Comput. 23 (1969), 23-28.

[9] Kitkuan, D. Kumam, P.; Martínez-Moreno, J. Generalized halpern-type forward-backward splitting methods for convex minimization problems with application to image restoration problems. Optimization 69 (2020), no. (7-8), 1557-1581.

[10] Lions, P. L.; Mercier, B. Splitting algorithms for the sum of two nonlinear operators. SIAM J. Numer. Anal. 16 (1979), no. 6, 964-979.

[11] Maingé, P. E. Convergence theorems for inertial KM-type algorithms. J. Comput. Appl. Math. 219 (2008), no. $1,223-236$.

[12] Martinet, B. Regularisation dinequations variationelles par approximations successives. Revue Fran Maise Dautomatique, Informatique, Recherche Opérationnelle Operationnelle (3) 4 (1970), no. 3, 154-158.

[13] Moudafi, A.; Al-Shemas, E. Simultaneous iterative methods for split equality problem. Trans. Math. Program. Appl. 1 (2013), 1-11.

[14] Moudafi, A.; M. Oliny, M. Convergence of a splitting inertial proximal method for monotone operators. J. Comput. Appl. Math. 155 (2003), no. 2, 447-454.

[15] Nakajo, K. Shimoji, K.; Takahashi, W. Strong convergence to common fixed points of families of nonexpansive mappings in Banach spaces. J. Nonlinear Convex Anal. 8 (2007), 11-34.

[16] Nakajo, K. Shimoji, K.; Takahashi, W. On strong convergence by the hybrid method for families of mappings in Hilbert spaces. Nonlinear Anal. Theor. Methods Appl. 71 (2009), 112-119.

[17] Peaceman, D. H.; Rachford, H. H. The numerical solution of parabolic and elliptic differential equations. SIAM J. Appl. Math. 3 (1955), 28-41.

[18] Polyak, B. T. Some methods of speeding up the convergence of iteration methods. USSR Comput. Math. $\mathcal{E}$ Math. Phys. 4 (1964), 1-17.

[19] Takahashi, W. Introduction to Nonlinear and Convex Analysis, Yokohama Publishers: Yokohama, Japan, 2009.

[20] Tibshirani, R. Regression shrinkage and selection via the lasso. J. R. Stat. Soc. B Methodol. 58 (1996), 267-288.

[21] Varga, R. S. Matrix Iterative Analysis, Springer-Verlag, New York, 2000.

[22] Verma, M.; Shukla, K. K. A new accelerated proximal gradient technique for regularized multitask learning framework. Pattern Recogn. Lett. 95 (2017), 98-103.

DEPARTMENT OF MATHEMATICS

FACULTY OF SCIENCE AND TECHNOLOGY

RAMBHAI BARNI RAJABHAT UNIVERSITY

CHANTHABURI 22000, THAILAND

E-mail address: anantachai.perbru.ac.th

E-mail address: or_duangkamon@hotmail.com 